\title{
Comparisons of CVID and IgGSD: Referring Physicians, Autoimmune Conditions, Pneumovax Reactivity, Immunoglobulin Levels, Blood Lymphocyte Subsets, and HLA-A and -B Typing in 432 Adult Index Patients
}

\author{
James C. Barton, ${ }^{1,2,3}$ Luigi F. Bertoli, ${ }^{1,2,4}$ and J. Clayborn Barton ${ }^{2}$ \\ ${ }^{1}$ Department of Medicine, Brookwood Medical Center, Suite 626, 2022 Brookwood Medical Center Drive, \\ Birmingham, AL 35209, USA \\ ${ }^{2}$ Southern Iron Disorders Center, Birmingham, AL 35209, USA \\ ${ }^{3}$ Department of Medicine, University of Alabama at Birmingham, Birmingham, AL 35294, USA \\ ${ }^{4}$ Brookwood Biomedical, Birmingham, AL 35209, USA \\ Correspondence should be addressed to James C. Barton; ironmd@isp.com
}

Received 30 May 2014; Revised 13 August 2014; Accepted 14 August 2014; Published 11 September 2014

Academic Editor: Roshini Sarah Abraham

Copyright (C) 2014 James C. Barton et al. This is an open access article distributed under the Creative Commons Attribution License, which permits unrestricted use, distribution, and reproduction in any medium, provided the original work is properly cited.

\begin{abstract}
Common variable immunodeficiency (CVID) and immunoglobulin (Ig) G subclass deficiency (IgGSD) are heterogeneous disorders characterized by respiratory tract infections, selective Ig isotype deficiencies, and impaired antibody responses to polysaccharide antigens. Using univariable analyses, we compared observations in 34 CVID and 398 IgGSD adult index patients (81.9\% women) referred to a hematology/oncology practice. Similarities included specialties of referring physicians, mean ages, proportions of women, reactivity to Pneumovax, median serum IgG3 and IgG4 levels, median blood CD56+/CD16+ lymphocyte levels, positivity for HLA-A and -B types, and frequencies of selected HLA-A, -B haplotypes. Dissimilarities included greater prevalence of autoimmune conditions, lower median IgG, IgA, and IgM, and lower median CD19+, CD3+/CD4+, and CD3+/CD8+ blood lymphocytes in CVID patients. Prevalence of Sjögren's syndrome and hypothyroidism was significantly greater in CVID patients. Combined subnormal IgG1/IgG3 occurred in 59\% and 29\% of CVID and IgGSD patients, respectively. Isolated subnormal IgG3 occurred in 121 IgGSD patients (88\% women). Logistic regression on CVID (versus IgGSD) revealed a significant positive association with autoimmune conditions and significant negative associations with IgG1, IgG3, and IgA and CD56+/CD16+ lymphocyte levels, but the odds ratio was increased for autoimmune conditions alone (6.9 (95\% CI 1.3, 35.5)).
\end{abstract}

\section{Introduction}

Common variable immunodeficiency (CVID) and immunoglobulin (Ig) G subclass deficiency (IgGSD) are clinically and genetically heterogeneous disorders characterized by recurrent or severe infections of the upper and lower respiratory tract or other sites, selective deficiencies of Ig isotypes, and impaired antibody responses to common bacterial polysaccharide and protein antigens. Some adults with CVID also have decreased numbers or function of blood lymphocyte subsets, autoimmune conditions, or chronic inflammation
[1-3]. Some adults with IgGSD have decreased numbers or function of blood lymphocyte subsets or autoimmune conditions [4-6]. Some human leukocyte antigen (HLA)-A and -B types and haplotypes (chromosome $6 \mathrm{p}$ ) are associated with increased risk for CVID and IgGSD in adults [7-11]. HLA-A and -B haplotypes $A^{*} 02, B^{*} 44$ and $A^{*} 03, B^{*} 07$ were associated with transmission of both CVID and IgGSD immunophenotypes in some kinships [10].

Thus, we evaluated clinical and laboratory features of 432 consecutive white adult CVID and IgGSD index patients (34 CVID, 398 IgGSD) referred to a single practice because 
they had frequent or severe respiratory tract infections. We compared age at diagnosis, sex, specialties of referring physicians, autoimmune conditions, levels of serum Ig isotypes, blood lymphocyte subset levels, and HLA-A and -B types and haplotypes of CVID and IgGSD patients. Our results are discussed in the context of previous reports of clinical and genetic features of CVID and IgGSD.

\section{Methods}

2.1. Patient Selection. The performance of this work was approved by the Institutional Review Board of Brookwood Medical Center. All patients reported herein were referred to a hematology/medical oncology practice for further evaluation and management because they had (a) frequent or severe infections uncontrolled by antibiotic therapy and other management and (b) evidence of hypogammaglobulinemia.

We defined CVID in accordance with the criteria of the Pan-American Group for Immunodeficiency and the European Society for Immunodeficiency [12]. In adults, these criteria include serum IgG and IgA levels at least 2 standard deviations (SD) below respective means for age; absent isohemagglutinins or poor response to vaccines; and exclusion of other defined causes of hypogammaglobulinemia [12].

There is no generally accepted definition of IgGSD. One authoritative source defined IgGSD as serum levels of one or more IgG subclasses (IgG1-3) at least 2 SD below the mean(s) for age in the presence of normal serum IgG, with or without low serum IgA [13]. We accepted that case definition but also included patients with low IgG and normal IgA as having IgGSD. Thus, each of the present patients diagnosed herein to have IgGSD had primary immunodeficiency with nonprotective serotype-specific serum IgG levels for or impaired responses to Streptococcus pneumoniae polysaccharide antigens.

We recommended that all patients accept vaccination with Pneumovax (Pneumovax 23, polyvalent pneumococcal vaccine; Merck, Sharpe \& Dohme, Whitehouse Station, NJ) at diagnosis of primary Ig deficiency. We measured pre- and post-Pneumovax Streptococcus pneumoniae serotype-specific IgG antibodies, as possible. Postvaccination antibody panels were measured at least 4 weeks after the initial vaccination and before IgG replacement therapy was initiated.

We performed a computerized and manual search of charts of all white adults ( $\geq 18$ years of age) in our practice who were referred as outpatients in the interval 1998-2013 because they had frequent or severe infections, typically of the upper and lower respiratory tract, and who were diagnosed to have CVID or IgGSD $[10,12,13]$. We designated the first persons in respective families diagnosed to have CVID or IgGSD as index patients. All patients resided in central Alabama.

We included observations on all index patients whose charts: (a) documented laboratory testing to establish their diagnosis of CVID or IgGSD, including flow cytometric analysis of blood lymphocyte subsets and HLA-A and -B typing and haplotyping and (b) contained a physician's recommendation that they be treated with either intravenous or subcutaneous IgG because they had frequent or severe infections. Autoimmune conditions were typically identified and characterized by referring physicians, our queries regarding autoimmune conditions, and medication reviews. Autoimmune conditions reported herein were diagnosed before diagnosis of CVID or IgGSD. We compiled histories of frequent or severe respiratory tract infections and autoimmune conditions in first-degree family members of each index patient.

2.2. Patient Exclusions. We excluded patients with (a) isolated subnormal IgA or IgM; (b) normal Ig levels with nonprotective serotype-specific IgG levels for Streptococcus pneumoniae polysaccharide antigen (s); (c) hypogammaglobulinemia attributed to B-cell neoplasms, organ transplantation, immunosuppressive therapy, anticancer chemotherapy, or increased Ig loss; (d) polyclonal or monoclonal gammopathy; and (e) human immunodeficiency virus infection. We also excluded persons of African American descent because (a) certain HLA types and haplotypes differ significantly among Caucasians and African Americans in central Alabama [1416]; (b) in adults, mean serum concentrations of Ig are often greater in persons of sub-Saharan African Native descent than in whites $[17,18]$; (c) persons of sub-Saharan African Native descent occur infrequently in series of CVID or IgGSD patients [1]; and (d) such patients are also uncommon in our experience.

2.3. Laboratory Methods. Testing was performed at diagnosis of CVID or IgGSD before IgG replacement therapy was initiated. Serum IgG, IgG subclasses, IgA, and $\operatorname{IgM}$ were measured using standard automated methods. We defined mean $\pm 2 \mathrm{SD}$ as the normal or reference range for all Ig measurements, consistent with other investigators [10,19-21]. Reference ranges are IgG 7.00-16.00 g/L; IgG1 4.22-12.92 g/L; IgG2 $1.17-7.47 \mathrm{~g} / \mathrm{L} ; \operatorname{IgG} 3 \quad 0.41-1.29 \mathrm{~g} / \mathrm{L} ; \operatorname{IgG} 40.01-2.91 \mathrm{~g} / \mathrm{L}$; IgA $0.70-4.00 \mathrm{~g} / \mathrm{L}$; and $\operatorname{IgM} 0.40-2.30 \mathrm{~g} / \mathrm{L}$. Subnormal Ig levels were defined as those below the corresponding lower reference limit. Elevated serum Ig levels were defined as those greater than the upper reference limit. Antinuclear antibody titers of $1: 80$ or greater unexplained by other conditions were defined as positive.

IgG antibody test panels included measurements of antibodies specific for 6,7 , or 14 S. pneumoniae serotypes, according to the year of diagnosis, physician choice, and insurance requirements. Six-serotype panels included these specificities: Types 1, 3, 14, 19, 23, and 51. Seven-serotype panels included these specificities: Types 4, 14, 19, 23, 26, 56, and 68. Fourteen-serotype panels included these specificities: Types 1, 3, 4, 8, 9, 12, 14, 19, 23, 26, 51, 56, 57, and 68 . We defined serotype-specific IgG levels as either protective $(\geq 1.3 \mathrm{mg} / \mathrm{L})$ or nonprotective $(<1.3 \mathrm{mg} / \mathrm{L})$ [22]. Responses to vaccinations were defined as (1) increments in the number of protective antibody levels after vaccination (dichotomous variable) and (2) percentage increments of the numbers of protective antibody levels in the postvaccination panel compared to those in the prevaccination panel (continuous variable).

Blood levels of lymphocyte subsets were measured using flow cytometry. Reference ranges (mean $\pm 2 \mathrm{SD}$ ) are 
CD19+ $12-645$ cells $/ \mu \mathrm{L} ; \quad$ CD3+/CD4+ 359-1,519 cells/ $\mu \mathrm{L}$; CD3+/CD8+ 109-897 cells/ $\mu \mathrm{L}$; and CD56+/CD16+ 24406 cells $/ \mu \mathrm{L}$. Subnormal levels were defined as those below the corresponding lower reference limit. Elevated subset levels were defined as those greater than the upper reference limit.

HLA-A and -B alleles were detected using low-resolution DNA-based typing (polymerase chain reaction/sequencespecific oligonucleotide probe) in index patients [10]. HLA typing of family members of some index patients was performed to permit assignment of chromosome $6 p$ haplotypes. In each index patient in whom a single $\mathrm{A}$ or $\mathrm{B}$ allele was detected by DNA-based typing, we verified the allele(s) and set phase to ascertain haplotypes of the index patient using HLA analyses of appropriate family members. Haplotypes were defined only by $\mathrm{A}$ and $\mathrm{B}$ alleles. We compiled all HLA-A and -B types in CVID and IgGSD patients. We also tabulated ten HLA-A and -B haplotypes that are associated with increased risk of CVID and IgGSD in central Alabama white index patients $[7,10]$.

2.4. Statistics. The final analytic data set consisted of observations on 432 index patients. Analyses were performed with Excel 2000 (Microsoft Corp., Redmond, WA) and GB-Stat (v. 10.0, 2003, Dynamic Microsystems, Inc., Silver Spring, MD). D'Agostino's test was used as a measure of normality. Descriptive data are displayed as enumerations, percentages, mean $\pm 1 \mathrm{SD}$, median (range), or mean (95\% confidence intervals (CI)). Age at diagnosis data were normally distributed and were compared using Student's $t$-test (two-tailed). Because measures of some serum Ig isotypes, some blood lymphocyte subsets, and percentages of protective antibody levels were not normally distributed, we compared these data using the Mann-Whitney $U$ test. Proportions were compared using Pearson's $\chi^{2}$ test or Fisher's exact test, as appropriate. Linear correlations were performed using Pearson's technique. We performed logistic regressions on CVID (CVID and IgGSD as dichhltotomous variables) using all available independent variables except IgG and HLA-A and -B types. We computed the odds ratios of the significant variables and deviance of the final regression model. A value of $P<0.05$ was defined as significant.

\section{Results}

3.1. General Characteristics of 432 Index Patients. There were 34 CVID patients and 398 IgGSD patients. The mean age at diagnosis of all patients was $49 \pm 13 \mathrm{y}$. Mean ages of CVID and IgGSD patients did not differ significantly (data not shown). Women comprised $81.9 \%$ of the entire cohort. The proportions of women in CVID and IgGSD patients did not differ significantly (data not shown). Primary care, rheumatology, or otolaryngology specialists referred $79.4 \%$ of all patients. Percentages of referring medical specialists did not differ significantly between CVID and IgGSD patients (Table 1). Frequent or severe respiratory tract infections in first-degree family members were reported by 8 CVID patients $(23.5 \%)$ and $153 \operatorname{IgGSD}$ patients $(38.4 \%)(P=0.0843)$. Autoimmune
TABLE 1: Medical specialists who referred 432 adult CVID/IgGSD index patients ${ }^{1}$.

\begin{tabular}{lccc}
\hline Referring physician & $\begin{array}{c}\text { CVID } \\
(n=34)\end{array}$ & $\begin{array}{c}\text { IgGSD } \\
(n=398)\end{array}$ & Value of $P$ \\
\hline Primary care, \% $(n)$ & $29.4(10)$ & $27.4(109)$ & 0.7998 \\
Rheumatology, \% (n) & $23.5(8)$ & $23.6(94)$ & 0.9907 \\
Otolaryngology, \% (n) & $20.6(7)$ & $28.9(115)$ & 0.3018 \\
Endocrinology, \% (n) & $8.8(3)$ & $3.0(12)$ & 0.1054 \\
Gastroenterology, \% (n) & $5.9(2)$ & $1.5(6)$ & 0.1248 \\
Pulmonology, \% $(n)$ & $5.9(2)$ & $11.8(47)$ & 0.2309 \\
Other $^{2}$ & $5.9(2)$ & $3.8(15)$ & 0.3031 \\
\hline
\end{tabular}

${ }^{1}$ CVID, common variable immunodeficiency; IgGSD, IgG subclass deficiency. Comparisons were made with Pearson's $\chi^{2}$ test or Fischer's exact test, as appropriate.

${ }^{2}$ Other: cardiology (2), gynecology (4), infectious disease (4), neurology (4), general surgery (2), and addictionology (1).

conditions in first-degree family members were reported by 4 CVID patients (17.8\%) and 82 IgGSD patients $(20.6 \%)(P=$ 0.2154).

3.2. Autoimmune Conditions. The prevalence of autoimmune conditions was greater in CVID patients ( $55.9 \%$ versus $36.9 \%$; $P=0.0292)$. In CVID and IgGSD patients with autoimmune conditions, $63.0 \%$ and $85.7 \%$ were women, respectively $(P=0.0045)$. Seven different autoimmune conditions were reported in CVID patients whereas 23 different autoimmune conditions were reported in IgGSD patients (Table 2). Sjögren's syndrome, Hashimoto's thyroiditis, and rheumatoid arthritis comprised $78.9 \%$ of autoimmune conditions in CVID patients and $39.4 \%$ of autoimmune conditions in IgGSD patients $(P<0.0001)$ (Table 2$)$. The prevalence of Sjögren's syndrome and Hashimoto's thyroiditis was significantly greater in CVID patients (Table 2). Hypothyroidism due to Hashimoto's thyroiditis or unreported cause occurred in $44.1 \%$ of CVID patients and $15.8 \%$ of IgGSD patients $(P<$ 0.0001) (Table 2).

3.3. Streptococcus pneumoniae Serotype-Specific IgG Antibodies. Panels of pre- and post-Pneumovax S. pneumoniae serotype-specific IgG antibodies were available in 28 CVID patients $(82.5 \%)$ and in $315 \operatorname{IgGSD}$ patients $(79.1 \%)(P=$ 0.6572 ). No CVID patient had pre- and post-Pneumovax antibody test panels in which $100 \%$ of serotype antibodies were "protective." In 26 IgGSD patients (8.3\%), pre-and postPneumovax antibody panels included $100 \%$ protective levels of serotype antibodies ( 0 versus $8.3 \%, P=0.1000)$. There was no increment in the number of protective IgG levels after Pneumovax in 7 CVID patients (25.0\%) and in 114 IgGSD patients $(36.2 \%)(P=0.2350)$.

We also evaluated the post-Pneumovax percentage increments of the numbers of protective antibody levels in patients whose protective pre-Pneumovax antibodies were less than $100 \%$. The median increment was $14.3 \%$ in 28 CVID patients and $14.3 \%$ in $289 \operatorname{IgGSD}$ patients $(P=0.5382$; Mann-Whitney $U$ test). 
TABLE 2: Autoimmune conditions in 432 adult CVID/IgGSD index patients ${ }^{1}$.

\begin{tabular}{|c|c|c|c|}
\hline Condition(s) & $\operatorname{CVID}(n=34)$ & $\operatorname{IgGSD}(n=398)$ & Value of $P$ \\
\hline Sjögren’s syndrome, \% $(n)$ & $20.6(7)$ & $6.5(26)$ & 0.0031 \\
\hline Hashimoto's thyroiditis, $\%(n)^{2}$ & $11.8(4)$ & $2.8(11)$ & 0.0059 \\
\hline Rheumatoid arthritis, \% (n) & $11.8(4)$ & $5.3(21)$ & 0.1199 \\
\hline Gastrointestinal tract, $\%(n)^{3}$ & $2.9(1)$ & $2.3(9)$ & 0.5634 \\
\hline Multiple sclerosis, \% (n) & $2.9(1)$ & $0.3(1)$ & 0.1514 \\
\hline Positive ANA, $\%(n)^{4}$ & $2.9(1)$ & $3.5(14)$ & 0.6664 \\
\hline Psoriatic arthritis, \% (n) & $2.9(1)$ & $1.3(5)$ & 0.3903 \\
\hline Autoimmune hearing loss, $\%(n)$ & 0 & $0.3(1)$ & 0.9213 \\
\hline Autoimmune hemolytic anemia, $\%(n)^{5}$ & 0 & $1.0(4)$ & 0.7196 \\
\hline Diabetes mellitus, type 1, \% ( $n)$ & 0 & $1.3(5)$ & 0.6624 \\
\hline Graves' disease, \% (n) & 0 & $0.5(2)$ & 0.7820 \\
\hline Inflammatory arthritis, $\%(n)^{6}$ & 0 & $0.8(3)$ & 0.7815 \\
\hline Interstitial cystitis, \% $(n)$ & 0 & $3.0(12)$ & 0.3690 \\
\hline Mixed connective tissue disorder, $\%(n)$ & 0 & $1.3(5)$ & 0.6624 \\
\hline Myasthenia gravis, $\%(n)$ & 0 & $1.0(4)$ & 0.7196 \\
\hline Polymyalgia rheumatica, \% ( $n$ ) & 0 & $0.3(1)$ & 0.9213 \\
\hline Raynaud's phenomenon, \% ( $n)$ & 0 & $1.8(7)$ & 0.5610 \\
\hline Reiter's uveitis, \% $(n)$ & 0 & $0.3(1)$ & 0.9213 \\
\hline Sarcoidosis, \% (n) & 0 & $0.3(1)$ & 0.9213 \\
\hline Skin and mucous membranes, $\%(n)^{7}$ & 0 & $2.3(9)$ & 0.4747 \\
\hline Systemic lupus erythematosus, \% (n) & 0 & $3.8(15)$ & 0.2863 \\
\hline Temporal arteritis, \% (n) & 0 & $0.5(2)$ & 0.7820 \\
\hline Thrombotic disorders, $\%(n)^{8}$ & 0 & $1.3(5)$ & 0.6624 \\
\hline
\end{tabular}

${ }^{1}$ CVID, common variable immunodeficiency; IgGSD, IgG subclass deficiency. These autoimmune conditions were diagnosed before referral for CVID/IgGSD evaluation. Comparisons were made with Pearson's $\chi^{2}$ test or Fischer's exact test, as appropriate. Some patients had two or more autoimmune conditions.

${ }^{2}$ Hypothyroidism of unreported cause was reported in 11 CVID patients $(32.4 \%)$ and $52 \operatorname{IgGSD}$ patients $(13.1 \%)(P=0.0022)$.

${ }^{3}$ Crohn's disease (3), ulcerative colitis (3), and pernicious anemia (3).

${ }^{4} \mathrm{ANA}$, antinuclear antibody. Titers of 1:80 or greater unexplained by other conditions were defined as positive.

${ }^{5}$ Warm-reacting IgG autoantibody.

${ }^{6}$ Arthritis interpreted as autoimmune but not otherwise specified.

${ }^{7}$ Cutaneous psoriasis (3), erythema nodosum (2), vitiligo (2), alopecia areata (1), and Behcet's syndrome (1).

${ }^{8}$ Antiphospholipid syndrome (2), anticardiolipin antibody (1), and lupus anticoagulant (2).

We compared positivity for HLA-A $* 01, \mathrm{~B} * 08$ in CVID and IgGSD patients who had autoimmune conditions. This haplotype is part of a common, highly conserved, multigene and major histocompatibility complex (MHC)-linked ancestral haplotype associated with increased risk of CVID, subnormal IgA, and diverse autoimmune conditions [23]. Positivity rates for HLA-A $* 01, \mathrm{~B} * 08$ in CVID and IgGSD patients did not differ significantly $(26.3 \%$ versus $18.4 \%$, respectively; $P=0.4085$ ).

3.4. Serum IgG, IgG Subclasses, IgA, and IgM. In CVID patients, distributions of IgG, IgG1, and IgG3 levels were normal. Other Ig levels were not normally distributed. In IgGSD patients, levels of all Ig isotypes were normally distributed. We determined correlations of IgG with the sum of IgG1-4 levels. In CVID patients, the Pearson correlation coefficient was $0.4766(P<0.0001)$. In IgGSD patients, the correlation coefficient was $0.7688(P<0.0001)$. In both CVID and IgGSD patients, the median IgG was slightly greater than the corresponding median of the sum of IgG1-4 ( $5.51 \mathrm{~g} / \mathrm{L}$ versus $5.26 \mathrm{~g} / \mathrm{L}, \mathrm{CVID} ; 7.58 \mathrm{~g} / \mathrm{L}$ versus $7.49 \mathrm{~g} / \mathrm{L}$, IgGSD).
Median levels of all Ig isotypes were significantly lower in CVID patients except IgG3 and IgG4 which did not differ significantly between CVID and IgGSD patients (Table 3 ). By definition, IgG was subnormal in all CVID patients. IgG was also subnormal in $135 \mathrm{IgGSD}$ patients (33.9\%). The respective prevalence of subnormal IgG3 and subnormal IgG4 in CVID and IgGSD patients did not differ significantly. The combination of subnormal IgG1/IgG3 occurred in 58.8\% of CVID patients and $28.9 \%$ of IgGSD patients $(P=0.0002)$.

Isolated subnormal IgG3 was detected in 121 of 398 IgGSD patients (30.4\%) among whom the proportion of women $(107 / 121 ; 88.4 \%)$ was higher than the proportion of women in IgGSD patients without isolated subnormal IgG3 (221/277; $79.8 \%)(P=0.0371)$. No CVID patients and few IgGSD patients had elevated IgG. No patient had elevated IgG4 (Table 3).

By definition, all CVID patients had subnormal IgA and thus the median IgA level was lower in CVID patients than IgGSD patients. IgA levels were also subnormal in $5.2 \%$ of IgGSD patients. The median IgM level was significantly lower in CVID patients. The proportion of CVID patients who had 
TABLE 3: Serum immunoglobulins in 432 adult CVID/IgGSD index patients ${ }^{1}$.

\begin{tabular}{|c|c|c|c|}
\hline Ig isotype & $\operatorname{CVID}(n=34)$ & $\operatorname{IgGSD}(n=398)$ & Value of $P$ \\
\hline Median IgG, g/L & $5.51(3.75,6.92)$ & $7.58(3.43,21.56)$ & $<0.0001$ \\
\hline Subnormal IgG, \% (n) & $100.0(34)$ & $33.9(135)$ & $<0.0001$ \\
\hline Elevated IgG, \% $(n)$ & 0 & $1.3(5)$ & 0.5109 \\
\hline Median IgG1, g/L & $3.24(2.42,4.37)$ & $4.10(0.95,14.60)$ & $<0.0001$ \\
\hline Subnormal IgG1, \% (n) & $97.1(38)$ & $56.3(228)$ & $<0.0001$ \\
\hline Elevated IgG1, \% (n) & 0 & $0.3(1)$ & 0.7698 \\
\hline Median IgG2, g/L & $1.34(0.17,3.50)$ & $2.51(0.31,16.50)$ & $<0.0001$ \\
\hline Subnormal IgG2, \% ( $n)$ & $35.3(12)$ & $8.8(35)$ & $<0.0001$ \\
\hline Elevated IgG2, \% $(n)$ & 0 & $0.3(1)$ & 0.7698 \\
\hline Median IgG3, g/L & $0.39(0.07,1.06)$ & $0.33(0.06,1.76)$ & 0.5821 \\
\hline Subnormal IgG3, \% (n) & $58.8(20)$ & $70.9(282)$ & 0.3336 \\
\hline Elevated IgG3, \% (n) & 0 & $1.5(6)$ & 0.4709 \\
\hline Median IgG4, g/L & $0.08(0,0.29)$ & $0.13(0,2.37)$ & 0.0008 \\
\hline Subnormal IgG4, \% (n) & $5.9(2)$ & $3.3(13)$ & 0.4239 \\
\hline Elevated IgG4, \% $(n)$ & 0 & 0 & - \\
\hline Median IgA, g/L & $0.56(0.04,0.69)$ & $1.55(0.04,5.55)$ & $<0.0001$ \\
\hline Subnormal IgA, \% (n) & $100.0(34)$ & $5.3(21)$ & $<0.0001$ \\
\hline Elevated IgA, \% (n) & 0 & $2.5(10)$ & 0.3497 \\
\hline Median IgM, g/L & $0.55(0.10,1.88)$ & $1.00(0.04,5.16)$ & $<0.0001$ \\
\hline Subnormal IgM, \% (n) & $26.5(9)$ & $13.6(54)$ & 0.0407 \\
\hline Elevated IgM, \% (n) & 0 & $7.3(29)$ & 0.1032 \\
\hline
\end{tabular}

${ }^{1}$ CVID, common variable immunodeficiency; IgGSD, IgG subclass deficiency; Ig, immunoglobulin; SD, standard deviation. Reference ranges (mean \pm 2 SD) are IgG 7.00-16.00 g/L; IgG1 4.22-12.92 g/L; IgG2 1.17-7.47 g/L; IgG3 0.41-1.29 g/L; IgG4 0.01-2.91 g/L; IgA 0.70-4.00 g/L; and IgM 0.40-2.30 g/L. Subnormal Ig levels were defined as those below the corresponding lower reference limit. Elevated serum Ig levels were defined as those greater than the upper reference limit. Serum Ig levels are expressed as median (range). Comparisons were made with Mann-Whitney $U$ test, Pearson's $\chi^{2}$ test, or Fisher's exact test, as appropriate.

subnormal IgM was also greater than that of IgGSD patients (Table 3).

3.5. Blood Lymphocytes. Levels of CD19+, CD3+/CD4+, CD3+/CD8+, and CD56+/CD16+ lymphocytes in both CVID and IgGSD patients were normally distributed, except CD3+/CD4+ lymphocytes in CVID patients. Median levels of CD19+, CD3+/CD4+, and CD3+/CD8+ lymphocytes were significantly lower in patients with CVID (Table 4). The proportions of CVID and IgGSD patients who had either subnormal or elevated CD19+ lymphocyte levels were low (Table 4).

Median levels of CD3+/CD4+ lymphocytes were significantly lower in CVID patients. Elevated CD3+/CD4+ lymphocyte levels occurred in $8.8 \%$ and $10.8 \%$ of CVID and IgGSD patients, respectively (Table 4). Median CD3+/CD8+ lymphocyte levels were significantly lower in CVID patients. Subnormal or elevated CD3+/CD8+ lymphocytes were uncommon in both CVID and IgGSD patients (Table 4). Median levels of CD56+/CD16+ lymphocytes did not differ significantly between CVID and IgGSD patients. Subnormal or elevated CD56+/CD16+ lymphocyte levels were uncommon in both CVID and IgGSD patients (Table 4).

3.6. HLA Types and Haplotypes. In CVID and IgGSD patients, positivity for HLA-A and -B types did not differ significantly (Tables 5 and 6). We also compared the frequencies of ten HLA-A and -B haplotypes selected because their respective frequencies were greater among Alabama CVID and IgGSD patients than in the general Alabama adult population [10]. In the present study, these haplotypes accounted for $47.1 \%$ of 68 chromosomes $6 \mathrm{p}$ in CVID patients and $37.6 \%$ of 796 chromosomes $6 \mathrm{p}$ in IgGSD patients $(P=$ 0.1221 ). The respective haplotype frequencies in CVID and IgGSD patients did not differ significantly (Table 7).

3.7. Logistic Regressions on CVID. With initial regressions on CVID (versus IgGSD), we excluded many of the available independent variables because they were not significant. Among these exclusions was reactivity to Pneumovax as either a dichotomous or continuous variable. A final regression model on CVID revealed significant positive association with autoimmunity $(P=0.0247)$ and significant negative associations with serum $\operatorname{IgG1}, \operatorname{IgG} 3$, and $\operatorname{IgA}(P<0.0001$, 0.0113 , and $<0.0001$, resp.) and CD56+/CD16+ blood lymphocyte levels $(P=0.0141)$. The odds ratio of these significant associations was increased only for autoimmunity $(6.9$ (95\% CI 1.3, 35.5)). This model accounted for $79 \%$ of the deviance in CVID occurrence.

\section{Discussion}

Similarities of 34 CVID and 398 IgGSD patients referred to a hematology/oncology practice determined using univariable 
TABLE 4: Blood lymphocytes in 432 adult CVID/IgGSD index patients ${ }^{1}$.

\begin{tabular}{|c|c|c|c|}
\hline Lymphocyte subset & CVID $(n=34)$ & $\operatorname{IgGSD}(n=398)$ & Value of $P$ \\
\hline Median CD19+ cells $/ \mu \mathrm{L}$ & $172(0,631)$ & $228(5,2340)$ & 0.0051 \\
\hline Subnormal CD19+ cells, $\%(n)$ & $5.9(2)$ & $0.5(2)$ & 0.0337 \\
\hline Elevated CD19+ cells, \% $(n)$ & 0 & $2.8(11)$ & 0.4014 \\
\hline Median CD3+/CD4+ cells $/ \mu \mathrm{L}$ & $783(57,1987)$ & $937(108,2984)$ & 0.0335 \\
\hline Subnormal CD3+/CD4+ cells, \% $(n)$ & $14.7(5)$ & $3.8(15)$ & 0.0147 \\
\hline Elevated CD3+/CD4+ cells, \% (n) & $8.8(3)$ & $10.8(43)$ & 0.4988 \\
\hline Median CD3+/CD8+ cells/ $\mu \mathrm{L}$ & $322(110,1304)$ & $431(48,2106)$ & 0.0453 \\
\hline Subnormal CD3+/CD8+ cells, \% $(n)$ & 0 & $2.0(8)$ & 0.5170 \\
\hline Elevated CD3+/CD8+ cells, \% (n) & 0 & $7.8(31)$ & 0.0715 \\
\hline CD56+/CD16+ cells/ $\mu \mathrm{L}$ & $118(39,295)$ & $146(4,626)$ & 0.1988 \\
\hline Subnormal CD56+/CD16+ cells, $\%(n)$ & 0 & $3.5(14)$ & 0.3116 \\
\hline Elevated CD56+/CD16+ cells, \% (n) & 0 & $1.8(7)$ & 0.5618 \\
\hline
\end{tabular}

${ }^{1}$ CVID, common variable immunodeficiency; IgGSD, IgG subclass deficiency; SD, standard deviation. Blood levels of lymphocyte subsets were measured using flow cytometry. Reference ranges (mean $\pm 2 \mathrm{SD}$ ) are CD19+ 12-645 cells $/ \mu \mathrm{L} ; \mathrm{CD} 3+/ \mathrm{CD} 4+359-1,519$ cells $/ \mu \mathrm{L}$; CD3+/CD8+ 109-897 cells/ $\mu \mathrm{L}$; and $\mathrm{CD} 56+/ \mathrm{CD} 16+24-406$ cells/ $\mu \mathrm{L}$. Subnormal levels were defined as those below the corresponding lower reference limit. Elevated subset levels were defined as those greater than the upper reference limit. Blood lymphocyte levels are expressed as median (range). Comparisons were made with Mann-Whitney $U$ test, Pearson's $\chi^{2}$ test, or Fisher's exact test, as appropriate.

TABLE 5: Positivity for HLA-A types in 432 adult CVID/IgGSD index patients ${ }^{1}$

\begin{tabular}{lccc}
\hline Type & CVID $(n=34)$ & IgGSD $(n=398)$ & Value of $P$ \\
\hline $\mathrm{A}^{*} 01$ & $38.2(13)$ & $31.4(125)$ & 0.4124 \\
$\mathrm{~A}^{*} 02$ & $52.9(18)$ & $51.1(203)$ & 0.8971 \\
$\mathrm{~A}^{*} 03$ & $26.5(9)$ & $25.1(100)$ & 0.8837 \\
$\mathrm{~A}^{*} 11$ & $2.9(1)$ & $9.6(38)$ & 0.1638 \\
$\mathrm{~A}^{*} 23$ & $2.9(1)$ & $3.8(15)$ & 0.6364 \\
$\mathrm{~A}^{*} 24$ & $11.8(4)$ & $15.8(63)$ & 0.0434 \\
$\mathrm{~A}^{*} 25$ & $5.9(2)$ & $3.8(15)$ & 0.3933 \\
$\mathrm{~A}^{*} 26$ & $5.9(2)$ & $4.8(19)$ & 0.5052 \\
$\mathrm{~A}^{*} 28$ & $2.9(1)$ & $0.8(3)$ & 0.2804 \\
$\mathrm{~A}^{*} 29$ & $2.9(1)$ & $8.8(35)$ & 0.2003 \\
$\mathrm{~A}^{*} 30$ & 0 & $4.8(19)$ & 0.2034 \\
$\mathrm{~A}^{*} 31$ & $5.9(2)$ & $4.3(17)$ & 0.4507 \\
$\mathrm{~A}^{*} 32$ & $8.8(3)$ & $6.0(24)$ & 0.5393 \\
$\mathrm{~A}^{*} 33$ & 0 & $2.0(8)$ & 0.5161 \\
$\mathrm{~A}^{*} 34$ & 0 & $0.5(2)$ & 0.8486 \\
$\mathrm{~A}^{*} 66$ & 0 & $0.8(3)$ & 0.7815 \\
\hline
\end{tabular}

${ }^{1}$ HLA, human leukocyte antigen; CVID, common variable immunodeficiency; IgGSD, IgG subclass deficiency. Results are displayed as \% $(n)$. Comparisons were made with Pearson's $\chi^{2}$ test or Fischer's exact test, as appropriate.

analyses included specialties of referring physicians, mean ages at diagnosis, proportions of women, reactivity to Pneumovax, median serum IgG3 and IgG4 levels, median levels of CD56+/CD16+ blood lymphocytes, positivity for HLA-A and -B types, and frequencies of selected HLA-A, -B haplotypes. Dissimilarities included greater prevalence of autoimmune conditions, and lower median serum $\operatorname{IgG}, \operatorname{IgA}$, and $\operatorname{IgM}$ levels and lower median levels of CD19+, CD3+/CD4+, and CD3+/CD8+ blood lymphocytes in CVID patients.
Our observations differ somewhat from those in other CVID (or IgGSD) case series reports. All of the present patients were adults and all were referred due to frequent or severe infections alone, although $56 \%$ of the present patients had one or more autoimmune conditions at CVID/IgGSD diagnosis. In 334 European CVID patients followed for an average of 26 years, $71 \%$ had one or more inflammatory/autoimmune complications and the remainder had infections only [2]. In 476 US CVID patients, one or more of these complications occurred in $68 \%$ of the patients whereas infections were the only manifestation in the remaining patients [24]. At diagnosis of CVID in 303 European adults, some patients had splenomegaly (45\%), lymphadenopathy (26\%), autoimmune hemocytopenias (20\%), or granulomatous disease (12\%) [25]. In two large series of European CVID patients, enteropathy, autoimmunity, granulomatous disease, and splenomegaly were common and formed a set of interrelated features $[25,26]$. Further, clinical phenotypes in CVID patients seem to be stable over long intervals [2, 24, 27]. Our informal observations suggest that enteropathy, granulomatous disease, and splenomegaly are uncommon in the present patients. Immune thrombocytopenia had not been diagnosed in any of the present patients before diagnosis of CVID or IgGSD, although this condition is the most prevalent autoimmune hemocytopenia in CVID patients [27-31].

There was a preponderance of women in both CVID and IgGSD patients in the present study, consistent with our previous report [10]. In studies of CVID, the proportions of men and women are typically equal $[1,12,27]$. In contrast, there is female predominance in adults with isolated subnormal IgG3 levels $[4,6,32]$. Likewise, the proportion of women among the present 121 IgGSD patients with isolated subnormal IgG3 was significantly greater than among IgGSD patients without isolated subnormal IgG3. Many of the present CVID and IgSD patients had autoimmune conditions. The prevalence of 
TABLE 6: Positivity for HLA-B types in 432 adult CVID/IgGSD index patients ${ }^{1}$.

\begin{tabular}{|c|c|c|c|}
\hline Type & $\operatorname{CVID}(n=34)$ & $\operatorname{IgGSD}(n=398)$ & Value of $P$ \\
\hline $\mathrm{B}^{*} 07$ & $29.4(10)$ & $22.9(91)$ & 0.3866 \\
\hline $\mathrm{B}^{*} 08$ & $38.2(13)$ & $25.4(101)$ & 0.1025 \\
\hline$B^{*} 13$ & 0 & $4.3(17)$ & 0.2414 \\
\hline $\mathrm{B}^{*} 14$ & $2.9(1)$ & $9.0(36)$ & 0.1874 \\
\hline $\mathrm{B}^{*} 15$ & $11.8(4)$ & $14.1(56)$ & 0.4757 \\
\hline $\mathrm{B}^{*} 18$ & $8.8(3)$ & $7.3(29)$ & 0.4708 \\
\hline $\mathrm{B}^{*} 27$ & $2.9(1)$ & $6.8(27)$ & 0.3322 \\
\hline$B^{*} 35$ & $8.8(3)$ & $14.1(56)$ & 0.2888 \\
\hline$B^{*} 37$ & $2.9(1)$ & $2.3(9)$ & 0.5634 \\
\hline $\mathrm{B}^{*} 38$ & $8.8(3)$ & $2.8(11)$ & 0.0891 \\
\hline$B^{*} 39$ & 0 & $3.5(14)$ & 0.1681 \\
\hline$B^{*} 40$ & $17.7(6)$ & $8.8(35)$ & 0.0894 \\
\hline$B^{*} 41$ & 0 & $1.0(4)$ & 0.7196 \\
\hline$B^{*} 44$ & $44.1(15)$ & $30.2(120)$ & 0.0917 \\
\hline$B^{*} 45$ & 0 & $2.3(9)$ & 0.4747 \\
\hline$B^{*} 47$ & 0 & $1.0(4)$ & 0.7196 \\
\hline$B^{*} 48$ & 0 & $0.3(1)$ & 0.9213 \\
\hline$B^{*} 49$ & 0 & $3.8(15)$ & 0.2863 \\
\hline$B^{*} 50$ & 0 & $2.8(11)$ & 0.3705 \\
\hline $\mathrm{B}^{*} 51$ & $14.7(5)$ & $8.3(33)$ & 0.1673 \\
\hline$B^{*} 52$ & 0 & $2.5(10)$ & 0.4366 \\
\hline $\mathrm{B}^{*} 53$ & $2.9(1)$ & $1.3(5)$ & 0.3903 \\
\hline$B^{*} 55$ & 0 & $3.5(14)$ & 0.3116 \\
\hline$B^{*} 56$ & 0 & $1.3(5)$ & 0.6624 \\
\hline$B^{*} 57$ & $2.9(1)$ & $7.0(28)$ & 0.3127 \\
\hline $\mathrm{B}^{*} 58$ & 0 & $0.5(2)$ & 0.8486 \\
\hline$B^{*} 60$ & 0 & $1.3(5)$ & 0.6624 \\
\hline$B^{*} 62$ & $2.9(1)$ & $0.5(2)$ & 0.2185 \\
\hline
\end{tabular}

${ }^{1}$ HLA, human leukocyte antigen; CVID, common variable immunodeficiency; IgGSD, IgG subclass deficiency. Results are displayed as \% (n). Comparisons were made with Pearson's $\chi^{2}$ test or Fischer's exact test, as appropriate.

autoimmune conditions is widely acknowledged to be greater in women [33]. Regardless, these observations only partially explain the predominance of women in the present cohort.

Autoimmune conditions had been diagnosed in $56 \%$ of the present CVID patients before their referral. Analyzing follow-up observations of patients for the diagnosis of other autoimmune conditions was beyond the scope of the present work. In other CVID case series, $12-46 \%$ of patients had autoimmune conditions [2, 24, 34-36]. Autoimmune conditions occurred in $37 \%$ of the present IgGSD patients and in $13 \%$ of adults with IgGSD in Spain [5]. Although the diversity of autoimmune conditions in the present IgGSD patients was greater than that of CVID patients, no CVID patient and only one IgGSD patient had hemocytopenia attributed to autoimmunity. This result differs from that of other CVID case series reports $[2,24,34-36]$. Autoimmunity in CVID patients with the more common phenotype characterized by infections may also differ from that of patients who have the less common phenotype associated with polyclonal
TABLE 7: Frequencies of HLA-A, -B haplotypes in 432 adult CVID/IgGSD index patients.

\begin{tabular}{lccc}
\hline Haplotypes & $\begin{array}{c}\text { CVID }(n=68 \\
\text { chromosomes })\end{array}$ & $\begin{array}{c}\text { IgGSD }(n=796 \\
\text { chromosomes })\end{array}$ & Value of $P$ \\
\hline $\mathrm{A}^{*} 01, \mathrm{~B}^{*} 08$ & $13.2(9)$ & $11.7(93)$ & 0.7034 \\
$\mathrm{~A}^{*} 02, \mathrm{~B}^{*} 44$ & $16.2(11)$ & $9.9(79)$ & 0.1053 \\
$\mathrm{~A}^{*} 02, \mathrm{~B}^{*} 62$ & $1.5(1)$ & $0.3(2)$ & 0.2182 \\
$\mathrm{~A}^{*} 02, \mathrm{~B}^{*} 60$ & $0.0(0)$ & $0.1(1)$ & 0.9213 \\
$\mathrm{~A}^{*} 03, \mathrm{~B}^{*} 07$ & $7.4(5)$ & $5.7(45)$ & 0.3569 \\
$\mathrm{~A}^{*} 03, \mathrm{~B}^{*} 14$ & $0.0(0)$ & $1.6(13)$ & 0.3418 \\
$\mathrm{~A}^{*} 03, \mathrm{~B}^{*} 44$ & $2.9(2)$ & $3.8(30)$ & 0.5314 \\
$\mathrm{~A}^{*} 29, \mathrm{~B}^{*} 44$ & $1.5(1)$ & $3.4(27)$ & 0.3370 \\
$\mathrm{~A}^{*} 31, \mathrm{~B}^{*} 40$ & $2.9(2)$ & $0.5(4)$ & 0.0745 \\
$\mathrm{~A}^{*} 32, \mathrm{~B}^{*} 14$ & $1.5(1)$ & $0.6(5)$ & 0.3894 \\
\hline
\end{tabular}

${ }^{1}$ HLA, human leukocyte antigen; CVID, common variable immunodeficiency; IgGSD, IgG subclass deficiency. These haplotypes were selected for study because their respective frequencies were significantly greater in Alabama CVID/IgGSD index patients than in population control subjects [10]. Results are displayed as \% $(n)$. Comparisons were made with Pearson's $\chi^{2}$ test or Fischer's exact test, as appropriate. These haplotypes accounted for $47.1 \%$ of 68 chromosomes $6 \mathrm{p}$ in CVID patients and $37.6 \%$ of 796 chromosomes $6 \mathrm{p}$ in IgGSD patients $(P=0.1221)$.

lymphocytic manifestations [24, 35]. Referral of almost onequarter of the present patients by rheumatologists could account in part for the predominance of Sjögren's syndrome in both CVID and IgGSD patients. Hypothyroidism of all causes occurred in $44 \%$ and $16 \%$ of the present CVID and IgGSD patients, respectively. In two other reports, the prevalence of hypothyroidism in CVID patients was $<1 \%$ [24] and $24 \%$ [37].

Median levels of CD19+ blood lymphocytes were significantly lower in CVID patients, but few had subnormal CD19+ blood lymphocytes, consistent with previous reports [27, 38]. Median blood levels of CD3+/CD4+ lymphocytes were also significantly lower in CVID patients. Subnormal $\mathrm{CD} 3+/ \mathrm{CD} 4+$ blood lymphocyte levels occurred in a minority of the present CVID patients, consistent with previous reports [39-41].

T-lymphocytes of some CVID patients express surface marker patterns indicative of chronic activation [42]. In contrast to $\mathrm{CD} 4+\mathrm{T}$-lymphocytes, numbers of $\mathrm{CD} 8+\mathrm{T}$ lymphocytes of these patients may increase, explaining the inverted $\mathrm{CD} 4 / \mathrm{CD} 8$ ratio reported in some patients with CVID [42]. CD8+ T-lymphocyte abnormalities have been associated with disturbed cytokine secretion [43], lower memory B-cell numbers and severe clinical courses [44], chronic or recurrent cytomegalovirus infections [45], and polyclonal expanded populations of large granular lymphocytes in association with splenomegaly [46]. Our informal observations suggest that CVID (or IgGSD) patients with these clinical attributes were uncommon in the present cohort, consistent with our observation that CVID patients had lower median blood CD3+/CD8+ lymphocyte levels and that no CVID patient had elevated blood CD3+/CD8+ lymphocyte levels. 
Median blood levels of CD56+/CD16+ lymphocytes did not differ significantly between the present CVID and IgGSD patients. Subnormal or elevated CD56+/CD16+ levels were uncommon in both CVID and IgGSD patients. In contrast, Aspalter and colleagues reported that absolute and relative blood natural killer lymphocyte (CD16+/CD56+) numbers measured by flow cytometry in CVID patients were low [47].

HLA-A* $01, B^{*} 08$ is linked to CVID, subnormal IgA levels, and a putative deleterious gene(s) in the HLA class II region $[7,8,11]$, presumably IGAD1 $[9,48]$. Thus, we had postulated that the frequency of haplotype $A^{*} 01, B^{*} 08$ would be greater in the present CVID patients (all of whom had subnormal IgA) than in IgGSD patients. Our postulate was not confirmed. In addition, proportions of the present CVID and IgGSD patients with autoimmune conditions who also had HLA-A* $01, B^{*} 08$ did not differ significantly. On the other hand, the respective frequencies of the ten selected haplotypes we studied were greater in patients with CVID or IgGSD than in the general Alabama adult population [10]. HLA-A and -B haplotypes $A^{*} 02, B^{*} 44$ and $A^{*} 03, B^{*} 07$ were associated with transmission of both CVID and IgGSD immunophenotypes in some kinships [10]. Thus, we infer that some of these haplotypes carry alleles that modulate blood Ig levels. Analysis of one family suggested linkage of CVID and subnormal IgA to chromosome 16q [49]. A genomewide association study demonstrated linkage of these traits to numerous other loci [50].

CVID phenotypes have been associated with autosomal recessive mutations in genes that encode proteins important for B-cell function. These proteins include inducible costimulatory (ICOS) [51], CD19 [52], B-cell activating factor receptor [53], CD20 [54], CD21 [55], and CD81 [56]. Deleterious mutations in the corresponding genes have been identified in a small number of CVID patients, some from consanguineous kinships [27]. Mutations in TACI occur in approximately $10 \%$ of CVID patients [27], but their presence is not diagnostic of CVID [57]. IgG3 is the most polymorphic human IgG subclass and includes thirteen G3m allotypes (variants of Ig heavy G chains) which constitute six major G3m alleles of IGHG3 on chromosome 14q32.33 [58]. Allotypes are characterized by differences in amino acid epitopes of the constant heavy $G$ chains and are inherited in a Mendelian manner [58-61]. Thus, alleles that would account for subnormal IgG3 in some CVID and IgGSD patients may be linked to chromosome 14q32.33. MRL/lpr mice carrying a congenic $\mathrm{H}$ $2^{\mathrm{b} / \mathrm{b}}$ MHC interval exhibit several abnormalities including very low IgG3 [62]. A genome-wide association study of 366 CVID patients from four geographic areas confirmed a strong association of CVID with the MHC and demonstrated many significant associations with other regions [63].

There are uncertainties regarding the present results. CVID (and possibly IgGSD) patients who had lymphadenopathy, splenomegaly, enteropathy, or granulomatous disease may not have been referred to our practice or were excluded by our present selection criteria. The predominance of women in our cohort is incompletely explained. The reason that the variety of autoimmune conditions is less in the present CVID than IgGSD patients is unknown, although it is unlikely that erroneous reporting of autoimmune conditions would account for the differences we observed. We did not tabulate reports of autoimmune conditions that occurred in index patients after diagnosis of CVID or IgGSD and thus the present estimates of the occurrence of autoimmune conditions are conservative. Paired pre- and post-Pneumovax S. pneumoniae serotype-specific antibody evaluations were unavailable in $21 \%$ of the present patients. Evaluations for absent isohemagglutinins or poor response to vaccines are incomplete in other large CVID case series [25]. It seems unlikely that our conclusions about responsiveness to pneumococcal polysaccharide antigens would have changed greatly had additional observations been available for analysis. Flow cytometry analysis of subsets of CD19+, $\mathrm{CD} 3+/ \mathrm{CD} 4+$, and $\mathrm{CD} 3+/ \mathrm{CD} 8+$ blood lymphocytes and functional studies of lymphocytes may have demonstrated additional similarities or dissimilarities between CVID and IgSD patients. Performing extended HLA haplotyping, analyzing genes associated with CVID, and Gm allotyping were also beyond the scope of the present work.

\section{Conclusions}

Using univariable analyses, we demonstrated similarities and dissimilarities of CVID and IgGSD patients. Logistic regression on CVID (versus IgGSD) revealed significant positive association with autoimmune conditions and significant negative associations with IgG1, IgG3, IgA, and CD56+/CD16+ blood lymphocyte levels, but the odds ratio was increased only for autoimmune conditions (6.9 (95\% CI 1.3, 35.5)).

\section{Conflict of Interests}

The authors declare that there is no conflict of interests regarding the publication of this paper.

\section{Acknowledgments}

This work was supported in part by Southern Iron Disorders Center and Brookwood Biomedical.

\section{References}

[1] C. Cunningham-Rundles and C. Bodian, "Common variable immunodeficiency: clinical and immunological features of 248 patients," Clinical Immunology, vol. 92, no. 1, pp. 34-48, 1999.

[2] H. Chapel, M. Lucas, M. Lee et al., "Common Variable immunodeficiency disorders: division into distinct clinical phenotypes," Blood, vol. 112, no. 2, pp. 277-286, 2008.

[3] J. H. Park, E. S. Resnick, and C. Cunningham-Rundles, "Perspectives on common variable immune deficiency," Annals of the New York Academy of Sciences, vol. 1246, no. 1, pp. 41-49, 2011.

[4] T. Söderström, R. Söderström, A. Avanzini, P. Brandtzaeg, G. Karlsson, and L. A. Hanson, "Immunoglobulin G subclass deficiencies," International Archives of Allergy and Applied Immunology, vol. 82, no. 3-4, pp. 476-480, 1987.

[5] E. Sarmiento, R. Mora, M. Rodríguez-Mahou, J. RodríguezMolina, E. Fernández-Cruz, and J. Carbone, "Autoimmune 
disease in primary antibody deficiencies," Allergologia et Immunopathologia, vol. 33, no. 2, pp. 69-73, 2005.

[6] F. Abrahamian, S. Agrawal, and S. Gupta, "Immunological and clinical profile of adult patients with selective immunoglobulin subclass deficiency: response to intravenous immunoglobulin therapy," Clinical and Experimental Immunology, vol. 159, no. 3, pp. 344-350, 2010.

[7] H. W. Schroeder Jr., Z. Zhu, R. E. March et al., "Susceptibility locus for IgA deficiency and common variable immunodeficiency in the HLA-DR3, -B8, -A1 haplotypes," Molecular Medicine, vol. 4, no. 2, pp. 72-86, 1998.

[8] C. A. Alper, D. Marcus-Bagley, Z. Awdeh et al., "Prospective analysis suggests susceptibility genes for deficiencies of IgA and several other immunoglobulins on the [HLA-B8, SC01, DR3] conserved extended haplotype," Tissue Antigens, vol. 56, no. 3, pp. 207-216, 2000.

[9] I. Vořechovský, M. Cullen, M. Carrington, L. Hammarström, and A. D. B. Webster, "Fine mapping of IGAD1 in IgA deficiency and common variable immunodeficiency: identification and characterization of haplotypes shared by affected members of 101 multiple-case families," Journal of Immunology, vol. 164, no. 8, pp. 4408-4416, 2000.

[10] J. C. Barton, L. F. Bertoli, and R. T. Acton, "HLA-A and -B alleles and haplotypes in 240 index patients with common variable immunodeficiency and selective IgG subclass deficiency in central Alabama," BMC Medical Genetics, vol. 4, article 3, 2003.

[11] J. Kralovicova, L. Hammarström, A. Plebani, A. D. B. Webster, and I. Vorechovsky, "Fine-scale mapping at IGAD1 and genome-wide genetic linkage analysis implicate HLA-DQ/DR as a major susceptibility locus in selective IgA deficiency and common variable immunodeficiency," Journal of Immunology, vol. 170, no. 5, pp. 2765-2775, 2003.

[12] M. E. Conley, L. D. Notarangelo, and A. Etzioni, "Diagnostic criteria for primary immunodeficiencies. Representing PAGID (Pan-American Group for Immunodeficiency) and ESID (European Society for Immunodeficiencies)," Clinical Immunology, vol. 93, no. 3, pp. 190-197, 1999.

[13] A. Aghamohammadi, V. Lougaris, A. Plebani, T. Miyawaki, A. Durandy, and L. Haaammarström, "Predominantly antibody deficiencies," in Primary Immunodeficiency Diseases, N. Rezaei, A. Aghamohammadi, and L. D. Notarangelo, Eds., chapter 3, pp. 97-130, Springer, Berlin, Germany, 2008.

[14] C. C. Murphy, R. T. Acton, B. O. Barger et al., "Population genetic analyses of insulin dependent diabetes mellitus using HLA allele frequencies," Clinical Genetics, vol. 23, no. 6, pp. 405414, 1983.

[15] R. T. Acton, L. Harman, R. C. P. Go, M.-L. Tseng, and W. Bias, "Comparison of HLA phenotypes among African Americans from Alabama, Maryland, and North Carolina," Transplantation Proceedings, vol. 25, no. 4, pp. 2404-2407, 1993.

[16] J. C. Barton, C. Q. Edwards, L. F. Bertoli, T. W. Shroyer, and S. L. Hudson, "Iron overload in African Americans," The American Journal of Medicine, vol. 99, no. 6, pp. 616-623, 1995.

[17] G. Shulman, G. C. Gilich, and M. J. A. Andrew, "Serum immunoglobulins G, A and M in White and Black adults on the Witwatersrand," South African Medical Journal, vol. 49, no. 29, pp. 1160-1164, 1975.

[18] M. Lewis, M. Miyashiro, J. Huton, L. Miller, and K. Sperber, "Immunoglobulin and IgG subclass levels in the African American and Hispanic populations of east Harlem," Mount Sinai Journal of Medicine, vol. 65, no. 2, pp. 139-145, 1998.
[19] S. O'Keeffe, A. Gzel, R. Drury, M. Cullina, J. Greally, and P. Finnegan, "Immunoglobulin G subclasses and spirometry in patients with chronic obstructive pulmonary disease," European Respiratory Journal, vol. 4, no. 8, pp. 932-936, 1991.

[20] S. L. Hill, J. L. Mitchell, D. Burnett, and R. A. Stockley, "IgG subclasses in the serum and sputum from patients with bronchiectasis," Thorax, vol. 53, no. 6, pp. 463-468, 1998.

[21] U. Schauer, F. Stemberg, C. H. L. Rieger et al., "IgG subclass concentrations in certified reference material 470 and reference values for children and adults determined with the binding site reagents," Clinical Chemistry, vol. 49, no. 11, pp. 1924-1929, 2003.

[22] J. S. Orange, M. Ballow, E. R. Stiehm et al., "Use and interpretation of diagnostic vaccination in primary immunodeficiency: a working group report of the Basic and Clinical Immunology Interest Section of the American Academy of Allergy, Asthma \& Immunology," Journal of Allergy and Clinical Immunology, vol. 130, no. 3, pp. S1-S24, 2012.

[23] P. Price, C. Witt, R. Allcock et al., "The genetic basis for the association of the 8.1 ancestral haplotype (A1, B8, DR3) with multiple immunopathological diseases," Immunological Reviews, vol. 167, pp. 257-274, 1999.

[24] E. S. Resnick, E. L. Moshier, J. H. Godbold, and C. CunninghamRundles, "Morbidity and mortality in common variable immune deficiency over 4 decades," Blood, vol. 119, no. 7, pp. 1650-1657, 2012.

[25] C. Wehr, T. Kivioja, C. Schmitt et al., "The EUROclass trial: defining subgroups in common variable immunodeficiency," Blood, vol. 111, no. 1, pp. 77-85, 2008.

[26] B. Gathmann, N. Mahlaoui, L. Gerard et al., "Clinical picture and treatment of 2212 patients with common variable immunodeficiency," Journal of Allergy \& Clinical Immunology, vol. 134, no. 1, pp. 116.el1-126.e11, 2014.

[27] C. Cunningham-Rundles, "The many faces of common variable immunodeficiency," Hematology, vol. 2012, pp. 301-305, 2012.

[28] M. Michel, V. Chanet, L. Galicier et al., "Autoimmune thrombocytopenic purpura and common variable immunodeficiency: analysis of 21 cases and review of the literature," Medicine, vol. 83, no. 4, pp. 254-263, 2004.

[29] A. K. Knight and C. Cunningham-Rundles, "Inflammatory and autoimmune complications of common variable immune deficiency," Autoimmunity Reviews, vol. 5, no. 2, pp. 156-159, 2006.

[30] D. Gobert, J. B. Bussel, C. Cunningham-Rundles et al., "Efficacy and safety of rituximab in common variable immunodeficiency-associated immune cytopenias: a retrospective multicentre study on 33 patients," British Journal of Haematology, vol. 155, no. 4, pp. 498-508, 2011.

[31] J. C. Podjasek and R. S. Abraham, "Autoimmune cytopenias in common variable immunodeficiency," Frontiers in Immunology, vol. 3, article 189, 2012.

[32] T. Söderström, R. Söderström, U. Bengtsson et al., "Clinical and immunological evaluation of patients low in single or multiple IgG subclasses," Monographs in Allergy, vol. 20, pp. 135-142, 1986.

[33] O. L. Quintero, M. J. Amador-Patarroyo, G. Montoya-Ortiz, A. Rojas-Villarraga, and J. Anaya, "Autoimmune disease and gender: Plausible mechanisms for the female predominance of autoimmunity," Journal of Autoimmunity, vol. 38, no. 2-3, pp. J109-J119, 2012.

[34] J. Boileau, G. Mouillot, L. Gérard et al., "Autoimmunity in common variable immunodeficiency: correlation with lymphocyte 
phenotype in the French DEFI study," Journal of Autoimmunity, vol. 36, no. 1, pp. 25-32, 2011.

[35] H. Abolhassani, D. Amirkashani, N. Parvaneh et al., "Autoimmune phenotype in patients with common variable immunodeficiency," Journal of Investigational Allergology and Clinical Immunology, vol. 23, no. 5, pp. 323-329, 2013.

[36] N. Ramírez-Vargas, S. E. Arablin-Oropeza, D. Mojica-Martínez et al., "Clinical and immunological features of common variable immunodeficiency in Mexican patients," Allergologia et Immunopathologia, vol. 42, no. 3, pp. 235-240, 2014.

[37] V. Bozoghlanian, S. Agrawal, and S. Gupta, "Comprehensive immunological and clinical features of common variable immunodeficiency (CVID)," Journal of Allergy \& Clinical Immunology, vol. 133, no. 2, supplement, pp. AB7-AB7, 2014.

[38] B. L. Ferry, J. Jones, E. A. Bateman et al., "Measurement of peripheral B cell subpopulations in common variable immunodeficiency (CVID) using a whole blood method," Clinical and Experimental Immunology, vol. 140, no. 3, pp. 532-539, 2005.

[39] M. J. de Vera, L. Al-Harthi, and A. T. Gewurz, "Assessing thymopoiesis in patients with common variable immunodeficiency as measured by T-cell receptor excision circles," Annals of Allergy, Asthma and Immunology, vol. 93, no. 5, pp. 478-484, 2004.

[40] A. Isgrò, M. Marziali, I. Mezzaroma et al., "Bone marrow clonogenic capability, cytokine production, and thymic output in patients with common variable immunodeficiency," Journal of Immunology, vol. 174, no. 8, pp. 5074-5081, 2005.

[41] A. Giovannetti, M. Pierdominici, F. Mazzetta et al., "Unravelling the complexity of $\mathrm{T}$ cell abnormalities in common variable immunodeficiency," Journal of Immunology, vol. 178, no. 6, pp. 3932-3943, 2007.

[42] U. Salzer, K. Warnatz, and H. H. Peter, "Common variable immunodeficiency-an update," Arthritis Research and Ther$a p y$, vol. 14, no. 5, article 223, 2012.

[43] A. M. Holm, E. A. Sivertsen, S. H. Tunheim et al., "Gene expression analysis of peripheral $\mathrm{T}$ cells in a subgroup of common variable immunodeficiency shows predominance of CCRT effector-memory T cells," Clinical and Experimental Immunology, vol. 138, no. 2, pp. 278-289, 2004.

[44] J. Viallard, P. Blanco, M. André et al., "CD8 ${ }^{+} H L A-D R^{+}$T lymphocytes are increased in common variable immunodeficiency patients with impaired memory B-cell differentiation," Clinical Immunology, vol. 119, no. 1, pp. 51-58, 2006.

[45] M. Raeiszadeh, J. Kopycinski, S. J. Paston et al., "The T cell response to persistent herpes virus infections in common variable immunodeficiency," Clinical and Experimental Immunology, vol. 146, no. 2, pp. 234-242, 2006.

[46] A. M. Holm, G. Tjønnfjord, A. Yndestad et al., "Polyclonal expansion of large granular lymphocytes in common variable immunodeficiency-association with neutropenia," Clinical and Experimental Immunology, vol. 144, no. 3, pp. 418-424, 2006.

[47] R. M. Aspalter, W. A. C. Sewell, K. Dolman, J. Farrant, and A. D. B. Webster, "Deficiency in circulating natural killer (NK) cell subsets in common variable immunodeficiency and X-linked agammaglobulinaemia," Clinical and Experimental Immunology, vol. 121, no. 3, pp. 506-514, 2000.

[48] J. E. Volanakis, Z. B. Zhu, F. M. Schaffer et al., "Major histocompatibility complex class III genes and susceptibility to immunoglobulin A deficiency and common variable immunodeficiency," Journal of Clinical Investigation, vol. 89, no. 6, pp. 1914-1922, 1992.
[49] A. A. Schäffer, J. Pfannstiel, A. D. B. Webster, A. Plebani, L. Hammarström, and B. Grimbacher, "Analysis of families with common variable immunodeficiency (CVID) and IgA deficiency suggests linkage of CVID to chromosome 16q," Human Genetics, vol. 118, no. 6, pp. 725-729, 2006.

[50] R. C. Ferreira, Q. Pan-Hammarström, R. R. Graham et al., "Association of IFIH1 and other autoimmunity risk alleles with selective IgA deficiency," Nature Genetics, vol. 42, no. 9, pp. 777780, 2010.

[51] B. Grimbacher, A. Hutloff, M. Schlesier et al., "Homozygous loss of ICOS is associated with adult-onset common variable immunodeficiency," Nature Immunology, vol. 4, no. 3, pp. 261268, 2003.

[52] H. Kanegane, K. Agematsu, T. Futatani et al., "Novel mutations in a Japanese patient with CD19 deficiency," Genes and Immunity, vol. 8, no. 8, pp. 663-670, 2007.

[53] K. Warnatz, U. Salzer, M. Rizzi et al., "B-cell activating factor receptor deficiency is associated with an adult-onset antibody deficiency syndrome in humans," Proceedings of the National Academy of Sciences of the United States of America, vol. 106, no. 33, pp. 13945-13950, 2009.

[54] T. W. Kuijpers, R. J. Bende, P. A. Baars et al., "CD20 deficiency in humans results in impaired T cell-independent antibody responses," Journal of Clinical Investigation, vol. 120, no. 1, pp. 214-222, 2010.

[55] J. Thiel, L. Kimmig, U. Salzer et al., "Genetic CD21 deficiency is associated with hypogammaglobulinemia," Journal of Allergy and Clinical Immunology, vol. 129, no. 3, pp. 801.e6-810.e6, 2012.

[56] M. C. van Zelm, J. Smet, B. Adams et al., "CD81 gene defect in humans disrupts CD19 complex formation and leads to antibody deficiency," Journal of Clinical Investigation, vol. 120, no. 4, pp. 1265-1274, 2010.

[57] Q. Pan-Hammarström, U. Salzer, L. Du et al., "Reexamining the role of TACI coding variants in common variable immunodeficiency and selective IgA deficiency," Nature Genetics, vol. 39, no. 4, pp. 429-430, 2007.

[58] C. Dechavanne, F. Guillonneau, G. Chiappetta et al., "Mass spectrometry detection of G3m and IGHG3 alleles and followup of differential mother and neonate IgG3," PLoS ONE, vol. 7, no. 9, Article ID e46097, 2012.

[59] A. Morell, F. Skvaril, A. G. Steinberg, E. van Loghem, and W. D. Terry, "Correlations between the concentrations of the four sub-classes of IgG and Gm Allotypes in normal human sera," The Journal of Immunology, vol. 108, no. 1, pp. 195-206, 1972.

[60] R. Grubb, T. Hallberg, L. Hammarstrom et al., "Correlation between deficiency of immunoglobulin subclass G3 and Gm allotype," Acta Pathologica Microbiologica et Immunologica Scandinavica C, vol. 94, no. 5, pp. 187-191, 1986.

[61] V. A. Oxelius, H. D. Ochs, and L. Hammarström, "Restricted immunoglobulin constant heavy $G$ chain genes in primary immunodeficiencies," Clinical Immunology, vol. 128, no. 2, pp. 190-198, 2008.

[62] H. Sekine, R. C. Ferreira, Q. Pan-Hammarström et al., "Role for Msh5 in the regulation of Ig class switch recombination," Proceedings of the National Academy of Sciences of the United States of America, vol. 104, no. 17, pp. 7193-7198, 2007.

[63] J. S. Orange, J. T. Glessner, E. Resnick et al., "Genomewide association identifies diverse causes of common variable immunodeficiency," Journal of Allergy and Clinical Immunology, vol. 127, no. 6, pp. 1360.e6-1367.e6, 2011. 


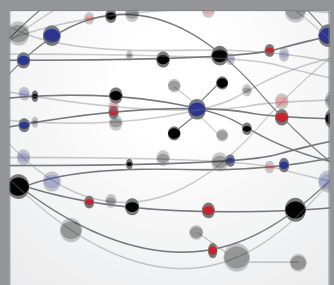

The Scientific World Journal
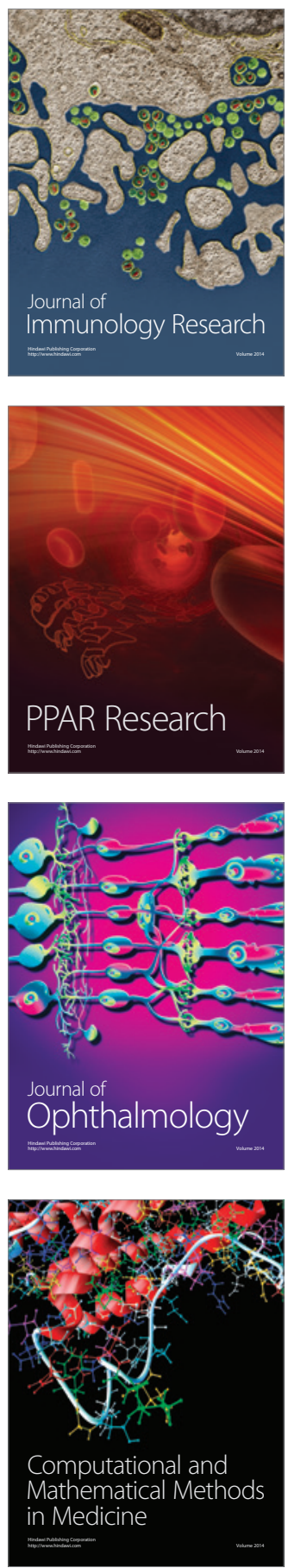

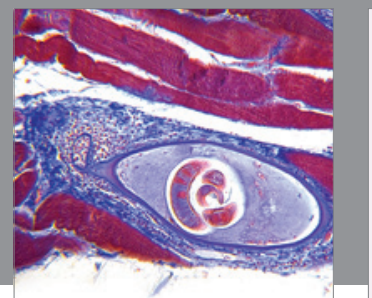

Gastroenterology

Research and Practice
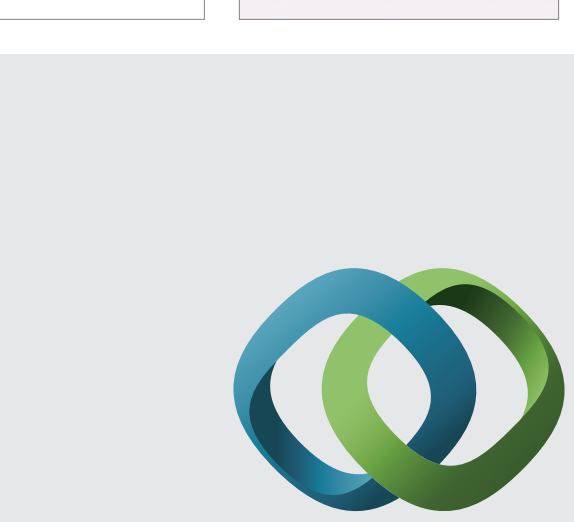

\section{Hindawi}

Submit your manuscripts at

http://www.hindawi.com
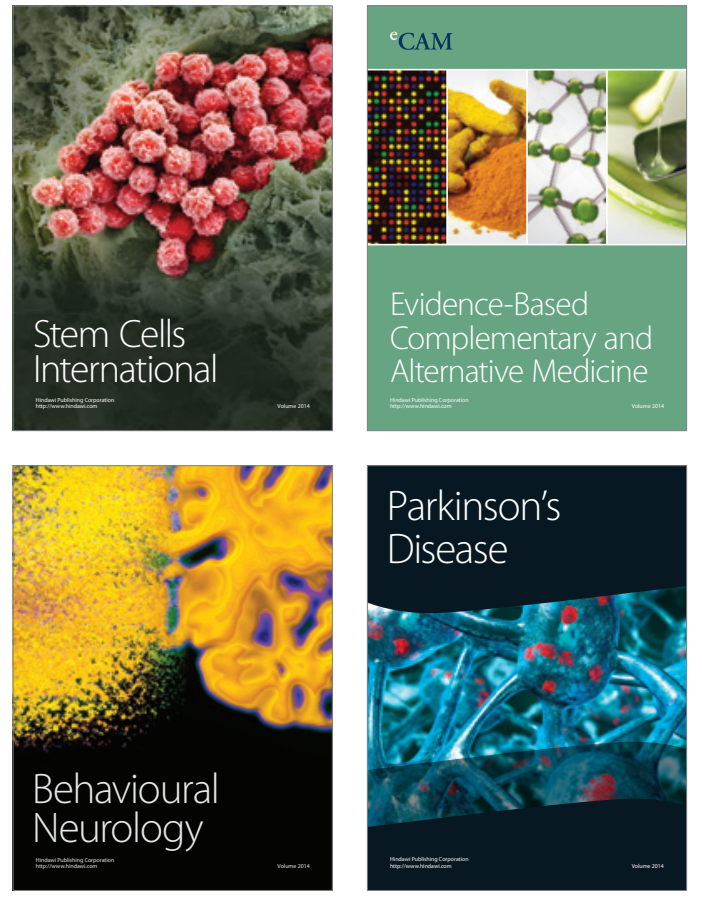
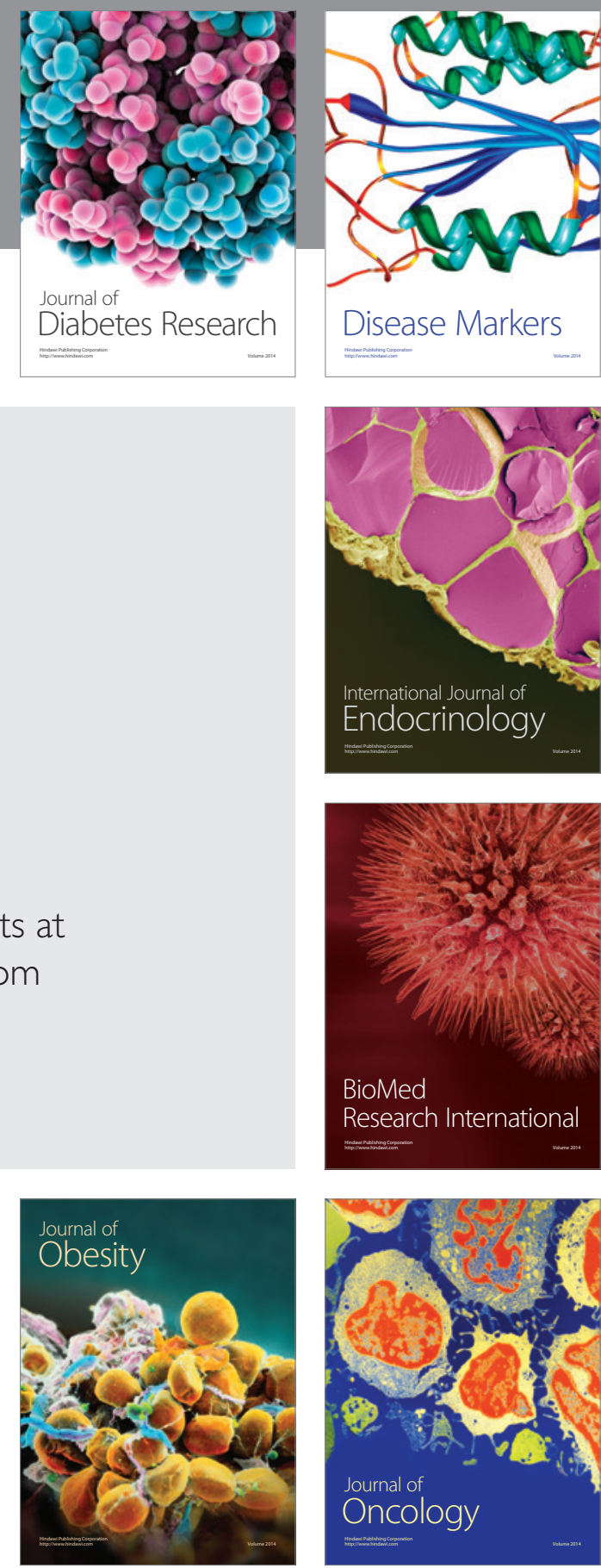

Disease Markers
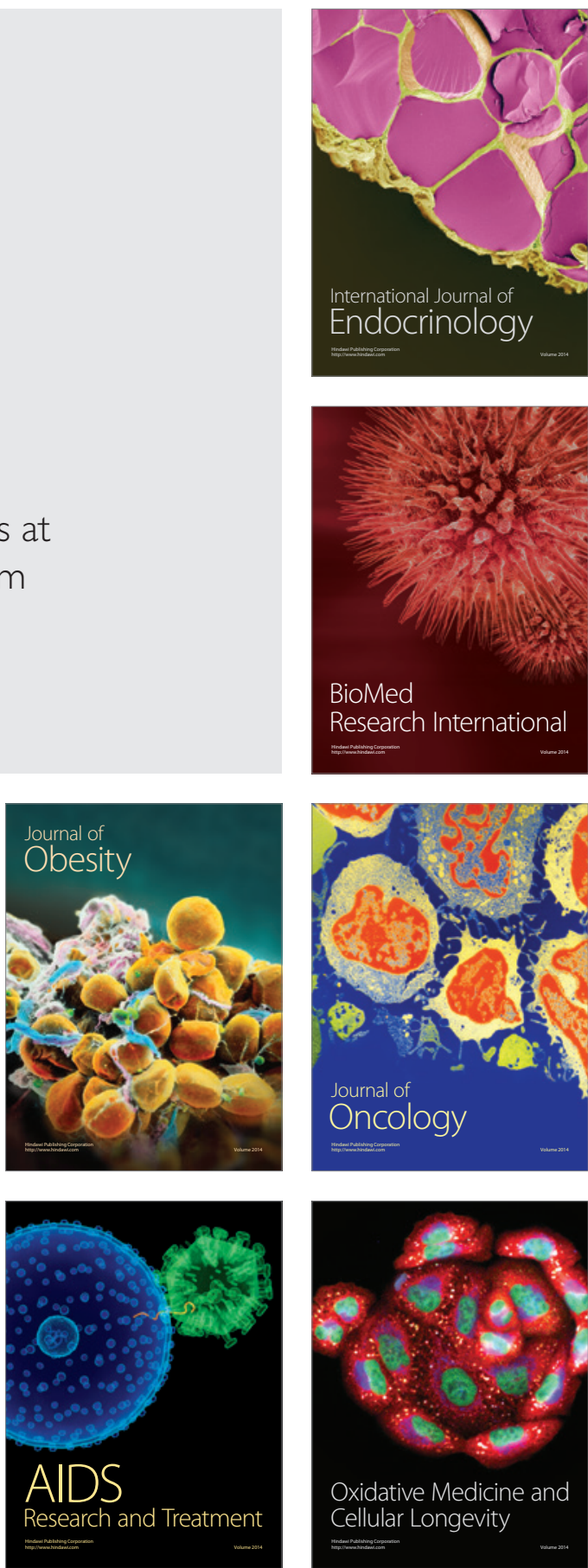